\title{
Modeling extracellular potentials
}

\author{
Gaute T. Einevoll • Daniel K. Wójcik • Alain Destexhe
}

Published online: 9 October 2010

(C) The Author(s) 2010. This article is published with open access at Springerlink.com

Recordings of extracellular potentials have been, and still are, a main workhorse in the quest for understanding how the brain works. The high-frequency part of the signal, the multi-unit activity (MUA), mainly reflects firing of action potentials in the vicinity of the electrode. The lowfrequency part, the local-field potential (LFP), appears largely to reflect subthreshold activity from a larger group of surrounding neurons. Despite their long history in neuroscience, these extracellular potentials are still not well understood. What can the LFP tell us about the underlying activity in neurons and neuronal networks? Which neurons dominate the MUA? These and similar questions need to be answered for us to take full advantage of the host of new types of multielectrodes with new geometries and ever more electrode contacts. Further, a better understanding of these issues will likely also be important for the development of good brain-machine interfaces. In all these problems, computational models and model-based analysis techniques are essential.

\section{G. T. Einevoll $(\bowtie)$}

Department of Mathematical Sciences and Technology and Center for Integrative Genetics, Norwegian University of Life Sciences, Ås, Norway

e-mail: Gaute.Einevoll@umb.no

D. K. Wójcik

Department of Neurophysiology,

Nencki Institute of Experimental Biology,

3 Pasteur St,

02-093 Warsaw, Poland

e-mail: d.wojcik@nencki.gov.pl

\section{A. Destexhe}

Integrative and Computational Neuroscience Unit (UNIC), UPR2191, CNRS,

Gif-sur-Yvette, France

e-mail: Destexhe@unic.cnrs-gif.fr
This was the backdrop and motivation for organizing the international workshop "Modeling and interpretation of extracellular potentials" at Ski, Norway on January 15th and 16th in 2009 (http://compneuro.umb.no/lfpworkshop/ LFP_workshop/). The main theme of the workshop was the development and use of new mathematical modeling and data analysis techniques to extract more information and insights from various types of multielectrode extracellular data. Supported by the Polish-Norwegian Research Fund, the workshop gathered about 70 researchers interested in these issues. In the final workshop discussion there seemed to be a consensus that stronger integrated computational and experimental research efforts are needed to fully utilize the new types of data offered by new multielectrodes. In particular one should seek to stimulate the development of a more closely connected research community focusing on such topics.

This special issue is a response to this request. Following an open call for papers in late January 2009 a total of 17 papers were in the end accepted and are included in the present issue. One of the important questions addressed is the biophysical bases of the LFP. While there is an agreement concerning the role of transmembrane currents as sources of the extracellular potentials, the filtering effects of the extracellular medium and its other effects on the recordings, as well as the effect of neuronal morphology, are still highly debated. These problems are addressed from different angles in the papers by Bangera, Schomer, Deghani, Ulbert, Cash, Papavasiliou, Eisenberg, Dale and Halgren "Experimental validation of the influence of white matter anisotropy of the intracranial EEG forward solution", by Bedard, Rodriques, Roy, Contreras and Destexhe in "Evidence for frequency-dependent extracellular impedance from the transfer function between extracellular and intracellular potentials", by Deghani, Bedard, Cash, 
Halgren and Destexhe in "Comparative power spectral analysis of simultaneous electroencephalographic and magnetoencephalographic recordings in humans suggests non-resistive extracellular media", and by Lindén, Pettersen and Einevoll in "Intrinsic dendritic filtering gives low-pass power spectra of local field potentials". These contributions examine the respective roles of dendritic filtering and factors such as frequencydependent extracellular impedances or tissue anisotropy in shaping the extracellular signatures of LFP signals.

Since the electric field is long range, any LFP recording may carry significant contributions from several populations of neurons. Thus, to understand the dynamics of the studied systems using LFP it is convenient to separate the signals into functional components, and two examples of the use of Independent Component Analysis for this purpose are included here. Makarov, Makarova and Herreras discuss the problem in the context of 1D recordings in the hippocampus in "Disentanglement of local field potential sources by independent component analysis", while Leski, Kublik, Swiejkowski, Wrobel and Wojcik in "Extracting functional components of neural dynamics with Independent Component Analysis and Inverse Current Source Density" show on three dimensional data from rat somatosensory thalamus how such analysis is facilitated by prior reconstruction of sources with the help of Inverse Current Source Density method. In "Encoding of brain state changes in local field potentials modulated by motor behaviors" Stamoulis and Richardson decompose cortical LFPs from rhesus monkeys using state-space methods into insignificant ongoing oscillations and meaningful LFP changes associated with the execution of movement.

If separation of components is not a viable solution one usually needs tailor-made methods or compare LFP recordings with results from mechanistic models mimicking the specific system at hand. This approach is taken in several papers aimed to explain specific features found in LFP: In "Cross-trial correlation analysis of evoked potentials reveals arousal-related attenuation of thalamocortical coupling" Sobolewski, Kublik, Swiejkowski, Leski, Kaminski and Wrobel present a new method for inferring functional connectivity from simultaneous LFP recordings and show how it can lead to new insights for thalamocortical recordings in rats. In "LFP Spectral Peaks in V1 Cortex: Network Resonance and Cortico-Cortical Feedback" Kang, Shelley, Henrie and Shapley consider a rate-based network model providing explanation for peaks observed in gamma band frequency spectra. Jedlicka, Deller and Schwarzacher have made a model of paired pulse inhibition of population spikes in dentate gyrus ("Computational modeling of GABAA receptor mediated paired-pulse inhibition in the dentate gyrus"). In "Spatial coherence and stationarity of local field potentials in an isolated whole hippocampal preparation in vitro" Gillis, Zhang and Skinner characterize the properties of LFP in hippocampal slices using first simulated data to calibrate their methods.

There is a lot of interest in investigating the relationship between local field potentials and simultaneously recorded spikes. This problem has been attacked from several perspectives in the present issue. The most direct approach is to use information theory and ask about the information content in the two signals. This approach is pursued in "Sensory information in local field potentials and spikes from visual and auditory cortices: time scales and frequency bands" by Belitski, Panzeri, Magri, Logothetis and Kayser, who study information content in spikes and different LFP bands. In "Causal relationships between frequency bands of extracellular signals in visual cortex revealed by an information theoretic analysis" Besserve, Scholkopf, Logothetis and Panzeri study causal relations between different frequency bands of LFP signals and MUA. An alternative to the information-theoretical approach is pursued in "Local field potentials indicate network state and account for neuronal response variability" by Kelly, Smith, Kass and Lee, who show how incorporation of the LFP signal in the point process model of spiking activity in V1 macaque monkeys improves the estimation of cell properties. Similarly, Galindo-Leon and Liu show that "Predicting stimulus-locked single unit spiking from cortical local field potentials" is possible for in vivo, awake, restrained mouse auditory cortical recordings.

In "Estimating the contribution of assembly activity to cortical dynamics from spike and population measures" Denker, Riehle, Diesmann and Grün describe methods to estimate spiking synchrony in neural assemblies from spike trains and LFP, illustrating their techniques with data from behaving rhesus monkeys performing motor tasks. Finally, Gawne studies "The local and non-local components for the local field potential in awake primate visual cortex" showing significant correlation between the evoked LFPs and the spiking activity recorded with the same electrode in V1.

As this brief overview indicates, at least three main directions for investigation of the theoretical underpinning and analysis of extracellular recordings are pursued today: One is the biophysical basis of the extracellular signals, that is exploration of different factors shaping the LFPs, such as biophysical mechanisms in the membrane, cell morphology, filtering properties of extracellular tissue, and so on. Secondly, the relation between spikes and LFPs recorded simultaneously is a topic of great interest. The degree of correlation between individual cell activity and the local and non-local population dynamics as reflected by the LFPs is crucial if we want to 
overcome the inevitable undersampling of individual neuron activity and is one of the situations where LFPs may be of great help. Finally, the complexity of spontaneous and evoked LFPs requires dedicated methods of analysis, in particular, the decomposition of the signals into functional components. These topics constitute important directions where computational neuroscience can make a decisive contribution, and we hope that this special issue will contribute to stimulating progress along all these research lines in the years to come.

Open Access This article is distributed under the terms of the Creative Commons Attribution Noncommercial License which permits any noncommercial use, distribution, and reproduction in any medium, provided the original author(s) and source are credited. 